\title{
Influence of baseline inducibility and activation mapping on ablation outcomes in patients with structural heart disease and ventricular tachycardia
}

\author{
Oscar Cano ${ }^{1}$, Víctor Pérez-Roselló ${ }^{1}$, Hebert Ayala ${ }^{1}$, Maite Izquierdo de Francisco ${ }^{1}$, Joaquín \\ Osca $^{1}, \mathrm{M}^{\mathrm{a}}$ José Sancho-Tello ${ }^{1}$, and Luis Martínez-Dolz ${ }^{1}$ \\ ${ }^{1}$ Hospital Politécnico y Universitario La Fe
}

February 15, 2021

\begin{abstract}
Introduction:Stand-alone substrate ablation without baseline ventricular tachycardia (VT) induction and activation mapping has become a standard VT ablation strategy. We sought to evaluate the influence of baseline VT inducibility and activation mapping on ablation outcomes in patients with structural heart disease (SHD) undergoing VT ablation. Methods:This is a single center, observational and retrospective study including consecutive patients with SHD and documented VT undergoing ablation. Baseline VT induction was attempted before ablation in all patients and VT activation mapping performed when possible. Ablation was guided by activation mapping for mappable VTs plus substrate ablation for all patients. Ablation outcomes and complications were evaluated. Results: 160 patients were included (203 VT ablation procedures) and were classified in 3 groups according to baseline VT inducibility: group 1 (non inducible, $\mathrm{n}=18$ ), group 2 ( 1 VT morphology induced, $\mathrm{n}=53$ ), and group 3 (>1VT morphology induced, $\mathrm{n}=89$ ). VT activation mapping was possible in $35 \%$. After a median follow-up of 38.5 months, baseline inducibility of $>1 \mathrm{VT}$ morphology was associated with a significant incidence of VT recurrence (42\% for group 3 vs. $15.1 \%$ for group 2 and $5.6 \%$ for group 1, Log-rank $\mathrm{p}<0.0001$ ) and activation mapping with a lower rate of VT recurrence $(24 \%$ vs. $36.3 \%$, Log-rank p=0.035). Independent predictors of VT recurrences and mortality were baseline inducibility of $>$ 1VT morphology (HR 12.05 IC 95\% 1.60-90.79, $\mathrm{p}=0.016$ ) and LVEF $<30 \%$ (HR 2.43 IC 95\% 1.45-4.07, p=0.001), respectively. Complications occurred in $11.2 \%$ (5.6\% hemodynamic decompensation). Conclusions:Baseline VT inducibility and activation mapping may add significant prognostic information during VT ablation procedur
\end{abstract}

Influence of baseline inducibility and activation mapping on ablation outcomes in patients with structural heart disease and ventricular tachycardia

Running title: Ventricular tachycardia ablation in structural heart disease

Óscar Cano ${ }^{1,2,3}$, MD, PhD, Víctor Pérez-Roselló ${ }^{1,2}$, MD, Hebert D. Ayala ${ }^{1,2}$, MD, Maite Izquierdo ${ }^{1,2}$, MD, $\mathrm{PhD}$, Joaquín Osca ${ }^{1,2}, \mathrm{MD}, \mathrm{PhD}$, María-José Sancho-Tello ${ }^{1,2}, \mathrm{MD}$, Luis Martínez-Dolz ${ }^{2,3}, \mathrm{MD}, \mathrm{PhD}$

${ }^{1}$ Arrhythmia Section, Department of Cardiology, Hospital Universitari i Politècnic La Fe, Valencia, Spain

${ }^{2}$ Instituto de Investigación Sanitaria La Fe, Valencia, Spain

${ }^{3}$ Centro de Investigaciones Biomédicas en RED en Enfermedades Cardiovasculares (CIBERCV), Spain.

Word count: 4982

\section{Conflict of interest}

Dr. O. Cano has received consultant fees from Biosense Webster. All other authors have nothing to disclose. 


\title{
Corresponding author:
}

Óscar Cano, MD, PhD

Hospital Universitari i Politècnic La Fe

Área de Enfermedades Cardiovasculares, Planta 4-Torre F

Av. Fernando Abril Martorell 106

46026, Valencia, Spain

Phone: +34961245849

Fax: +34961246238

E-mail:cano_osc@gva.es

\begin{abstract}
Introduction : Stand-alone substrate ablation without baseline ventricular tachycardia (VT) induction and activation mapping has become a standard VT ablation strategy. We sought to evaluate the influence of baseline VT inducibility and activation mapping on ablation outcomes in patients with structural heart disease (SHD) undergoing VT ablation.
\end{abstract}

Methods : This is a single center, observational and retrospective study including consecutive patients with SHD and documented VT undergoing ablation. Baseline VT induction was attempted before ablation in all patients and VT activation mapping performed when possible. Ablation was guided by activation mapping for mappable VTs plus substrate ablation for all patients. Ablation outcomes and complications were evaluated.

Results : 160 patients were included (203 VT ablation procedures) and were classified in 3 groups according to baseline VT inducibility: group 1 (non inducible, $\mathrm{n}=18$ ), group 2 (1 VT morphology induced, $\mathrm{n}=53$ ), and group 3 ( $>1 \mathrm{VT}$ morphology induced, $\mathrm{n}=89$ ). VT activation mapping was possible in $35 \%$. After a median follow-up of 38.5 months, baseline inducibility of $>1 \mathrm{VT}$ morphology was associated with a significant incidence of VT recurrence ( $42 \%$ for group 3 vs. $15.1 \%$ for group 2 and $5.6 \%$ for group 1, Log-rank p $<0.0001$ ) and activation mapping with a lower rate of VT recurrence $(24 \%$ vs. $36.3 \%$, Log-rank $\mathrm{p}=0.035)$. Independent predictors of VT recurrences and mortality were baseline inducibility of $>1 \mathrm{VT}$ morphology (HR 12.05 IC $95 \%$ 1.60-90.79, $\mathrm{p}=0.016$ ) and $\mathrm{LVEF}<30 \%$ (HR 2.43 IC 95\% 1.45-4.07, $\mathrm{p}=0.001$ ), respectively. Complications occurred in $11.2 \%$ ( $5.6 \%$ hemodynamic decompensation).

Conclusions : Baseline VT inducibility and activation mapping may add significant prognostic information during VT ablation procedures.

Key words: ventricular tachycardia; ablation; activation mapping; substrate mapping

\section{Introduction}

Ventricular tachycardia (VT) ablation strategies in patients with structural heart disease (SHD) have significantly evolved during the last years. Substrate ablation with complete elimination of the potentially arrhythmogenic substrate has become a standard treatment in this setting and has been related to better acute success rates and reduction of VT recurrences during follow-up ${ }^{1-8}$. This strategy is usually preferred for patients with hemodynamyic instability during induced VT, advanced heart failure with poor hemodynamic condition or non-inducible patients at the time of the procedure. However, stand-alone substrate ablation has also been advocated as a first ablation strategy even in the absence of these previous conditions thus obviating the evaluation of baseline VT inducibility and the potential utility of VT activation mapping ${ }^{9-13}$. We sought to evaluate the role of baseline VT inducibility and the influence of activation mapping on outcomes in patients with SHD undergoing VT ablation.

\section{Methods}


This is a single-center, observational retrospective study including consecutive patients with SHD and documented sustained monomorphic VT (SMVT) referred to our center for VT ablation. All patients undergoing VT ablation in our center are prospectively included in a dedicated database. Baseline clinical, imaging and analitics data as well as procedural and follow-up details are included in this database. Patients were included in the final data analysis only if a baseline programmed ventricular stimulation (PVS) protocol aiming for VT induction was performed after substrate mapping and before ablation. All patients gave informed consent and the study was carried out following the recommendations of the ettical committee of the Hospital Universitari i Politècnic La Fe.

\section{Workflow}

The procedures were performed either under general anesthesia or under concious sedation with propofol and fentanyl. Endocardial LV mapping was preferentially performed by a transseptal access although the retroaortic approach was also used when considered necessary. For those cases in which an epicardial involvement was suspected, an epicardial access was obtained using the technique described by Sosa et al. ${ }^{14}$

A complete substrate mapping of the chamber/s of interest was initially obtained during the baseline patient's rhythm and using either a linear (Smart-Touch, Biosense Webster) or a multielectrode catheter (PentaRay, Biosense Webster). Low voltage areas as well as late potentials were registered and tagged. Scar was defined as areas with bipolar voltage $<0.5 \mathrm{mV}$. Upon completion of the substrate mapping, a standardized PVS protocol was used to induce VT in all patients included in the study. This protocol consisted in a 8 beats basal train at three different cycle lengths $(600,500$ and $400 \mathrm{~ms})$ followed by up to 4 extraestimmuli with a minimun coupling interval of $200 \mathrm{~ms}$ or until ventricular refractoriness from the right ventricular apex, right ventricular outflow tract, or different LV sites. If a stable and hemodynamically tolerated SMVT was induced, activation mapping of the VT was performed and entrainment maneuvers were used whenever possible to clearly define the VT mechanism and circuit. In case of hemodynamic inestability VT was paced terminated or cardioverted. In this last scenario, the 12-lead ECG QRS morphology of the induced tachycardia was used as a reference during pacemapping. When the initially induced VT was mapped and/or terminated (spontaneously, pace-terminated, shocked or during radiofrequency ablation), PVS was performed again aiming for induction of additional VTs.

Once the areas of interest were identified, a contact force ablation catheter (Smart Touch, Biosense Webster) was used for radiofrequency (RF) delivery. In patients with a hemodynamically stable VT, RF was applied during tachycardia to evaluate the response to RF ablation. In case of VT interruption during RF application, additional lessions were administered to eliminate the substrate and then a new PVS protocol was used to test VT inducibility. In the case of non-inducibility and presence of abnormal signals (late potentials) additional lessions were applied until all the abnormal signals were abolished. Meanwhile, in all patients with hemodynamically unstable VT a substrate ablation strategy ( \pm pacemapping as an adjunctive strategy whenever possible) was performed targeting all the sites with abnormal signals registered during the initial substrate mapping. Patients with baseline non-inducibility underwent substrate ablation exclusively.

\section{Objectives}

The primary objective of the study was to evaluate the influence of baseline VT inducibility and VT activation mapping on patient's outcome in terms of VT recurrence during follow-up.

Secondary objectives included the evaluation of baseline VT inducibility and VT activation mapping influence on the developement of hemodynamic inestability during or after the ablation procedure and on mortality/heart transplantation during follow-up.

\section{Definitions and variables}

The clinical tachycardia was defined as the tachycardia previously registered in a 12-lead ECG or, when 12-lead ECG was not available, the tachycardia registered in the ICD log in terms of cycle length and/or intracardiac electrogram morphology. Abnormal electrograms included late potentials registered during the patient's baseline rhythm defined as a high frequency low amplitud signals occurring in the terminal part 
of the QRS complex (last $40 \mathrm{~ms}$ ) or beyond QRS complex termination and diastolic or presystolic potentias registered during VT.

Complete activation mapping was considered when the critical isthmus and or exit of the tachycardia circuit could be clearly defined by entrainment maneuvers. Acute success was defined as non-inducibility of any type of sustained ventricular arrhythmias at the end of the procedure. Survival free of VT recurrence was defined as the absence of sustained VT, symptomatic or asymptomatic, either registered by intracardiac electrograms from an ICD or from a surface 12 lead ECG.

We also recorded the incidence of new-onset of hemodynamic inestability during the procedure or during the first 24-48 h after ablation and the possible relationship with VT induction and the use of activation mapping during the procedure. Hemodynamic inestability during the procedure was defined as persistent hypotension despite vasopressors and requiring mechanical support or procedure discontinuation. During the first 24-48h after the procedure any hemodynamic worsenig, the need of vasoactive drugs or ventricular assist device implantation was also cosidered as procedure-related hemodynamic decompensation.

\section{Follow-up}

All patients included had a clinical follow-up after the procedure until hospital discharge. Then, patients were followed in the arrhythmia clinic every 6 months and whenever considered necessary. All patients with a previous ICD were included in a remote monitoring program.

Statistical Analysis

Data are presented as mean \pm standard deviation or median and interquartile range (IQR) for continuous variables. Discrete variables are presented as percentage and were compared using Chi-square test while continuous variables were compared using either the t- Student test or analysis of varianze (ANOVA) as appropriate. VT recurrence free survival was calculated using the Kaplan-Meier method with differences between groups compared with the log-rank test. Time-to-event was defined as time from procedure to occurrence of outcome event. Death from any cause within the follow-up period was considered for mortality analysis and was censored at date of death for VT recurrence endpoint. To identify predictors of VT recurrence and death univariate and multivariate Cox proportional hazards models were used. For multivariate analysis, variables traditionally associated with VT recurrences and with a $\mathrm{P}$ value [?] 0.15 in the univariate analysis were included in the model. HR and $95 \%$ confidence intervals (CI) from the Cox model were reported. Two-tailed $\mathrm{P}$ values $<0.05$ were considered statistically significant. All analyses were performed by using SPSS (IBM SPSS Statistics, Version 22.0, Armonk, New York, USA).

\section{Results}

A total of 203 VT ablation procedures were performed in 163 patients with SHD in our institution from March 2011 to September 2019. In 3 patients PVS was not performed before ablation due to baseline hemodynamic inestability and were not included in the study. The remaining 160 patients were finally included in the analysis and were distributed according to the results of baseline PVS (figure 1): group 1 (not inducible patients) $\mathrm{n}=18$, group 2 ( 1 VT morphology induced) $\mathrm{n}=53$, and group 3 ( $>1$ VT morphology induced), $\mathrm{n}=89$. In 4 patients the procedure was performed under mechanical hemodynamic support (ECMO in 3 patients and Levithronix in 1 patient). Baseline characteristics of the patients are described in table 1. Table 2 shows the principal findings during the VT ablation procedure.

\section{Effect of baseline VT inducibility on acute success and VT recurrences}

PVS was performed at the end of the procedure in 129 patients (80.6\%). In the remaining 31 patients PVS was not performed at the discretion of the operator because of perceived risk of hemodynamic inestabilization or previous baseline non-inducibility. Overall acute success rate of the procedure in patients with baseline VT inducibility (groups 2 and 3) and considering non-inducibility of any sustained VT was $66 \%$ ( $76 \%$ for group 2 and $60 \%$ for group $3, \mathrm{p}=0.068)$. The clinical VT was still inducible in $6.2 \%$ of patients ( $4.5 \%$ and 
$7.7 \%$ for groups 2 and 3, respectively) and any non-clinical VT/pleomorphic/polymorphic VT or VF was present in $17.8 \%$ of patients at the end of the procedure ( $4.5 \%$ and $26.9 \%$ for groups 2 and 3, respectively).

Overall survival free of VT recurrence after a single procedure was $71,1 \%$ with a median follow-up of 38.5 months (IQR 16.8-63.2). There were significant differences in survival free of VT recurrence among groups depending on baseline VT inducibility: $94.4 \%$ for group 1; $84.9 \%$ for group 2 and $58 \%$ for group 3, Logrank $\mathrm{p}<0.0001$ for overall comparison, Log-rank $\mathrm{p}=0.001$ for comparison between group 2 and 3 (figure $2 \mathrm{~A}$ ). Repeat procedures were performed in 31 patients (mean $1.25+-0.58$ procedures per patient) with an overall survival free of VT recurrence that increased to $85.6 \%$ after the last procedure with a median follow-up of 29.5 months (IQR 38.9-54.4). Group 1 survival free of VT recurrence after the last procedure remained stable at $94.4 \%$ but increased for patients in group 2 (94.3\%) and group $3(78.7 \%)$.

\section{Effect of activation mapping on acute success and VT recurrences}

Complete activation mapping was performed in 50 patients $(35.2 \%, 50 / 142$ inducible patients) and was significantly more frequently among patients with only 1 VT morphology induced compared with patients with $>1$ VT morphology induced $(49.1 \%$ vs. $27 \%, \mathrm{p}=0.011)$. In the remaining 110 patients substrate guide ablation +- pacemapping was used as ablation strategy. Acute success was comparable between patients with complete activation mapping and patients without activation mapping $(81.2 \%$ vs $70.7 \%$, respectively, $\mathrm{p}=0.22)$.

However, survival free of VT recurrence was significantly higher when activation mapping had been performed during the procedure ( $76 \%$ vs $63 \%$, Log-rank $\mathrm{p}=0.028$ )(figure $2 \mathrm{~B}$ ). Of note, in patients with only 1 VT induced (group 2) and complete activation mapping (26 group 2 patients) only 4 VT recurrences occurred during follow-up in comparison with $44 \mathrm{VT}$ recurrences in 115 patients with either $>1 \mathrm{VT}$ induced or impossibility of activation mapping (84.6\% survival free of VT recurrence vs. 63.8\%, respectively, Log-rank $\mathrm{p}=0.002)$ (Supplementary material).

\section{Predictors of VT recurrence during follow-up}

Inducibility of $>1$ VT morphology, the presence of a large endo/epicardial scar $(>30 \%)$, LVEF $<30 \%$ and impossibility of performing activation mapping during VT ablation were associated with VT recurrence in univariate analysis (table 3). A Cox proportional model was used for multivariate analysis and variables with a p value [?]0.15 in univariate analysis were included in the model. Baseline induction of $>1$ VT morphology was the only independent predictor of VT recurrence in multivariate analysis (HR 12.05 IC 95\% 1.60-90.79, $\mathrm{p}=0.016)$ (table 3).

Mortality

Overall mortality was $35.6 \%$ (50\%, 30.2\% and $36 \%$ for group 1, 2 and 3, respectively) without significant differences between groups (Log-rank $\mathrm{p}=0.398)$ (figure $4 \mathrm{~B}$ ). Causes of death were classified as arrhythmic/sudden death in 5 cases (8.8\%), advanced heart failure in 27 cases $(47.4 \%)$, non-cardiac deaths in 17 cases $(29.8 \%)$ and unknown cause in 8 cases $(14 \%)$. VT recurrence was not associated with mortality/cardiac transplantation (mortality/transplant $51 \%$ for patients with VT recurrence vs. $38.9 \%$ for patients without VT recurrence, Log-rank $\mathrm{p}=0.253$ ). However, patients with baseline $1 \mathrm{VT}$ morphology (group 2) and activation mapping during the procedure had significantly lower mortality during follow-up $(19.2 \%$ vs $38.8 \%$, Logrank $\mathrm{p}=0.039$ )(figure $4 \mathrm{~A}$ ). In multivariate analysis the only independent predictor of mortality was baseline LVEF $<30 \%$ (HR 2.43 CI 95\% 1.45-4.07, $\mathrm{p}<0.001$ )(table 4).

\section{Procedure related complications}

Overall complications rate was $11.3 \%$ without significant differences among groups $(11.1 \%$ for group 1, $17 \%$ for group 2 and $7.9 \%$ for group $3, \mathrm{p}=0.251$ )(table 2). Complications included: 4 cardiac tamponades requiring pericardiocentesis, 3 arterial pseudoaneurysms (2 requiring surgical correction) and 1 femoral hematoma not requiring further interventions. Finally 1 patient died after an acute coronary oclussion (proximal LAD) during the procedure which was inmediately stented but resulting in refractary cardiogenic shock. 
Only 9 patients (5.6\%) had an acute hemodynamic decompensation during or after the VT ablation procedure. Baseline mean PAINSDD score was 10.7+-6.7 (<10: 48.8\%, 10-16: $31.2 \%$ and [?]17: 20\%). There was no relationship between acute hemodynamic decompensation and activation mapping. In patients with inducible VT (groups 2 and 3), only 1/50 patients (2\%) with activation mapping developed acute hemodynamic decompensation in comparison with 6/92 patients with VT inducibility but no activation mapping, $\mathrm{p}=0.421$. The incidence of acute hemodynamic decompensation depending on the PAINSDD score was as follows: $6.4 \%$ for PAISDD $<10.2 \%$ for PAINSDD 10-16, and $9.4 \%$ for PAINSDD [?]17, $\mathrm{p}=0.337$. None of group 1 patients had acute hemodynamic decompensation in comparison with $7.5 \%$ of group 2 patients and $5.6 \%$ of group 3 patients, $\mathrm{p}=0.486$. Of these 9 patients, 2 died due to refractary cardiogenic shock (one of them after a ventricular assist device was implanted during the ablation procedure) and 1 underwent cardiac transplantation while in the rest 6 patients the hemodynamic decompensation was managed with inotropic drugs and could be stabilized and recovered without further complications.

\section{Discussion}

\section{Principal findings}

The main finding of our study is that baseline VT inducibility is independently associated with VT recurrences during follow-up in patients with SHD undergoing VT ablation. Thus, patients with no baseline VT inducibility or only 1 VT morphology induced had a significantly higher survival free of VT recurrences when compared with patients with more than $1 \mathrm{VT}$ morphology induced. In the same manner, complete activation mapping during the VT ablation procedure allowing delinitation of the VT circuit was associated with lower VT recurrences. Of note, although no overall relationship between baseline VT inducibility and mortality could be stablished, the combination of inducibility of a single VT morphology and VT activation mapping during the ablation procedure was associated with lower mortality.

\section{Utility of baseline inducibility and activation mapping during VT ablation}

VT in the setting of SHD is usually associated with the presence of scar and the principal eletrophysiological mechanism involved in the genesis and manteinance of the tachycardias is re-entry. Traditional VT ablation strategies involved baseline induction of the tachycardia and activation mapping when possible trying to depict the tachycardia circuit in order to guide the ablation strategy. During the last years substrate mapping and ablation without baseline VT induction or activation mapping has proved to be an effective and safe strategy ${ }^{9-13}$. The rationale for this new approach is that complete elimination of the abnormal substrate/signals is associated with better acute success in terms of non-inducibility of any VT (either clinical or not) at the end of the procedure when compared with ablation of the clinical VT only, and has clearly been related with a significant reduction of VT recurrences during follow-up. On the other hand, concerns regarding the developement of hemodynamic decompensation during or after VT ablation procedures have also favored the avoidance of baseline VT induction and activation mapping ${ }^{15-16}$. However, one of the downsides of stand-alone substrate ablation is that it may lead to an extensive ablation that may not be always necessary and could be avoided by a more physiologic approach identifying and ablating only the critical parts of the substrate that are responsible of VT generation and maintenance. Moreover, although re-entry is the principal mechanism of scar-related VT, other less frequent VT mechanisms can be present in patients with SHD and would not be identified unless VT induction is attempted thus hindering the procedure success probabilities ${ }^{17-18}$.

Current existing literature in this regard is controversial ${ }^{11-13}$. Two previous meta-analyses have evaluated the influence of VT ablation strategy on outcome. Briceno et al. included 396 patients from 6 different studies (5 observational cohort studies and 1 randomized prospective study) comparing complete substrate ablation vs. standard ablation of stable $\mathrm{VTs}^{12}$. Substrate ablation was found to be associated with a decrease in the composite end point of ventricular arrhythmias recurrence/all-cause mortality when compared with the standard approach (RR 0.57, 95\% CI 0.40-0.81). Kumar et al. included 403 patients from 6 studies and found no differences in the VT recurrence rate between substrate based VT ablation vs. standard VT ablation using activation mapping/entrainment with a mean follow-up of 18 months $(0.72,95 \%$ confidence 
interval [CI] 0.44-1.18, $\mathrm{p}=0.2)^{13}$.

Our study shows that baseline VT inducibility adds significantly important prognostic information in patients with SHD undergoing VT ablation. Following this strategy, patients with baseline inducibility of $>1 \mathrm{VT}$ morphology are clearly identified at a very high risk of VT recurrences (42\% over median follow-up of 38.5 months) while patients with no baseline inducibility or with only 1 VT morphology induced have very low VT recurrences rates (6.6\% and $15.1 \%$ after the first procedure). It is remarkable that PVS at the end of the procedure did not have this prognostic value in our series. Group 2 patients with acute success at the end of the procedure (determined by non inducibility with PVS) had significantly higher VT recurrence free survival when compared with group 3 patients with acute success $(85.4 \%$ vs. $63.6 \%$, Log-rank $\mathrm{p}=0.005)$ (Supplementary data).

Moreover, activation mapping during VT seems to add additional value and was also associated with lower VT recurrence rates in our study although did not reach statistical significance in multivariate analysis. The utility of VT activation mapping has been recently described ${ }^{19-20}$. Hadjis et. al. ${ }^{19}$ showed that VT recurrence was significantly lower among those patients with complete delineation of the diastolic pathway (12\%) during VT when compared with partial or no recording of the diastolic pathway (50\% and $45 \%$, respectively, $\mathrm{p}=0.02)$.

Of interest, hemodynamic decompensation during or after the procedure occurred only in 9 patients $(5.6 \%)$ in our series. This is a low percentage considering the presence of baseline VT inducibility in up to $89 \%$ of the patients and complete VT activation mapping in 35\%. Santangeli et al. reported $11 \%$ incidence of acute hemodynamic decompensation among 193 patients with scar-related VT undergoing VT ablation ${ }^{15}$. Unfortunately, no information about the incidence of acute hemodynamic decompensation depending on the ablation strategy has been yet published.

\section{Limitations}

This is a single center observational study with the inherent limmitations to this study design and using the specific strategy described so the results can not be extrapolated to other VT ablation protocols. Further randomized studies comparing substrate ablation vs. baseline VT induction + activation mapping when possible + critical isthmus substrate ablation should be encouraged to definitively elucidate the role of baseline VT inducibility and activation mapping in this setting. A significant percentage of patients was on active treatment with antiarrhythmic drugs at the time of the procedure and this could have influenced the results of baseline PVS.

\section{Conclusions}

Baseline VT inducibility of $>1$ VT morphology during VT ablation in patients with SHD is independently associated with a significant increase in VT recurrence during follow-up. Baseline VT inducibility and activation maping may add significant prognostic information in patients with SHD undergoing VT ablation.

\section{Founding sources: none}

\section{Conflict of interest}

Dr. O. Cano has received consultant fees from Biosense Webster and Boston Scientific. All other authors have nothing to disclose.

\section{References}

1. Arenal A, Glez-Torrecilla E, Ortiz M, et al. Ablation of electrograms with an isolated, delayed component as treatment of unmappable monomorphic ventricular tachycardia in patients with structural heart disease. J Am Coll Cardiol. 2003;41:81-92.

2. Jais P, Maury P, Khairy P, et al. Elimination of local abnormal ventricular activities: a new end point for substrate modification in patients with scar-related ventricular tachycardia. Circulation. 2012;125:21842196. 
3. Komatsu Y, Daly M, Sacher F, et al. Electrophysiologic characterization of local abnormal ventricular activities in postinfarction ventricular tachycardia with respect to their anatomic location. Heart Rhythm. 2013;10:1630-1637.

4. Di Biase L, Santangeli P, Burkhardt DJ, et al. Endo-epicardial homogenization of scar versus limited substrate ablation for the treatment of electrical storms in patients with ischemic cardiomyopathy. J Am Coll Cardiol. 2012;60:132-141.

5. Gokoğlan Y, Mohanty S, Gianni C, et al. Scar homogenization versus limited-substrate ablation in patients with nonischemic cardiomyopathy and ventricular tachycardia. J Am Coll Cardiol. 2016;68:1990-1998.

6. Tzou WS, Frankel DS, Hegeman T, et al. Core isolation of critical arrhythmia elements for treatment of multiple scar-mediated ventricular tachycardias. Circ Arrhythm Electrophysiol. 2015;8:353-361.

7. Berruezo A, Fernández-Armenta J, Andreu D, et al. Scar dechanneling: new method for scar-related left ventricular tachycardia substrate ablation. Circ Arrhythm Electrophysiol. 2015;8:326-36.

8. Tilz RR, Makimoto H, Lin T, et al. Electrical isolation of a substrate after myocardial infarction: a novel ablation strategy for unmappable ventricular tachycardias-feasibility and clinical outcome. Europace 2014;16:1040-52.

9. Fernández-Armenta J, Penela D, Acosta J, et al. Substrate modification or ventricular tachycardia induction, mapping, and ablation as the first step? A randomized study. Heart Rhythm. 2016;13:1589-1595.

10. Wolf M, Sacher F, Cochet H, Kitamura T, et al. Long-Term Outcome of Substrate Modification in Ablation of Post-Myocardial Infarction Ventricular Tachycardia. Circ Arrhythm Electrophysiol. 2018;11:e005635.

11. Breitenstein A, Sawhney V, Providencia R, et al. Ventricular tachycardia ablation in structural heart disease: Impact of ablation strategy and non-inducibility as an end-point on long term outcome. Int J Cardiol. 2019 Feb 15;277:110-117.

12. Briceño DF, Romero J, Villablanca PA, et al. Long-term outcomes of different ablation strategies for ventricular tachycardia in patients with structural heart disease: systemic review and meta-analysis. Europace. 2018;20:104-115.

13. Kumar S, Baldinger SH, Romero J, et al. Substrate-based ablation versus ablation guided by activation and entrainment mapping for ventricular tachycardia: A systematic review and meta-analysis. J Cardiovasc Electrophysiol. 2016;27:1437-1447.

14. Sosa E, Scanavacca M, d'Avila A, Pilleggi F. A new technique to perform epicardial mapping in the electrophysiology laboratory. J Cardiovasc Electrophysiol. 1996;7:531-6.

15. Santangeli P, Muser D, Zado ES, et al. Acute hemodynamic decompensation during catheter ablation of scar-related ventricular tachycardia: incidence, predictors, and impact on mortality. Circ Arrhythm Electrophysiol. 2015;8:68-75.

16. Muser D, Castro SA, Liang JJ, Santangeli P. Identifying risk and management of acute haemodynamic decompensation during catheter ablation of ventricular tachycardia. Arrhythmia \& Electrophysiology Review 2018;7:282-7.

17. Bogun F, Good E, Reich S, et al. Role of Purkinje fibers in post-infarction

ventricular tachycardia. J Am Coll Cardiol 2006;48:2500-2507.

18. Soejima K, Stevenson WG, Sapp JL, Selwyn AP, Couper G, Epstein LM. Endocardial and epicardial radiofrequency ablation of ventricular tachycardia associated with dilated cardiomyopathy: the importance of low-voltage scars

J Am Coll Cardiol $2004 ; 43: 1834-1842$. 
19. Hadjis A, Frontera A, Limite LR, et al. Complete electroanatomic imaging of the diastolic pathway is associated with improved freedom from ventricular tachycardia recurrence. Circ Arrhythm Electrophysiol. 2020;13:e008651.

20. Bourier F, Martin R, Martin CA, et al. Is it feasible to offer 'targeted ablation' of ventricular tachycardia circuits with better understanding of isthmus anatomy and conduction characteristics? Europace. 2019;21:i27i33.

\section{Figure Legends.}

Figure 1: Flowchart of patients included in the study.

Figure 2: A. Cumulative survival free of VT recurrence stratified by baseline inducibility over 66 months; B. Cumulative survival free of VT recurrence stratified by activation mapping during VT ablation procedure over 66 months.

Figure 3: A. Cumulative survival free of death/cardiac transplantation stratified by baseline inducibility + VT activation mapping over 66 months; B. Cumulative survival free of death/cardiac transplantation stratified by VT recurrence over 66 months.

Figure 4. Principal outcomes per study group (\%).

\section{Figure 1.}

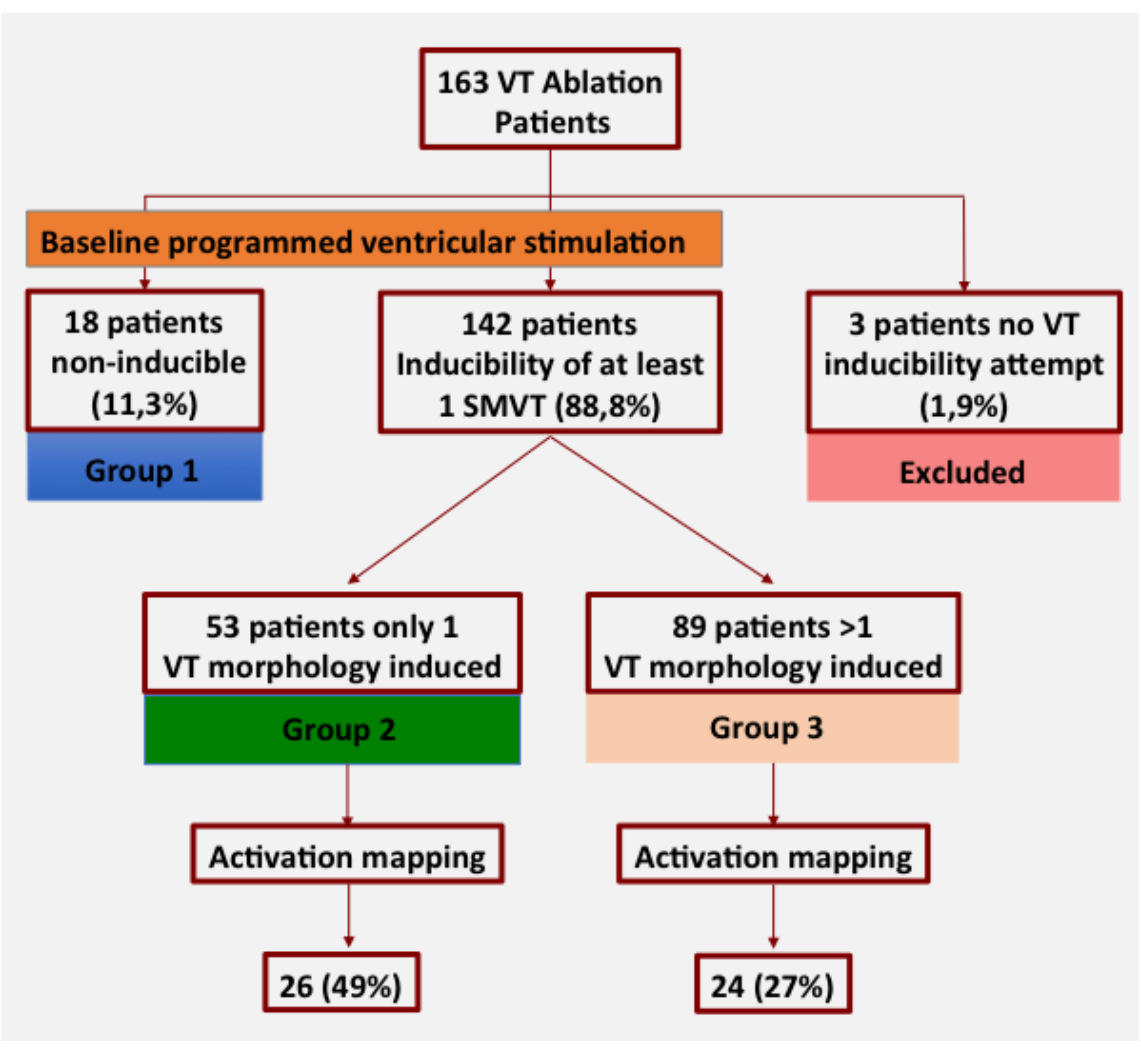

Figure 2. 

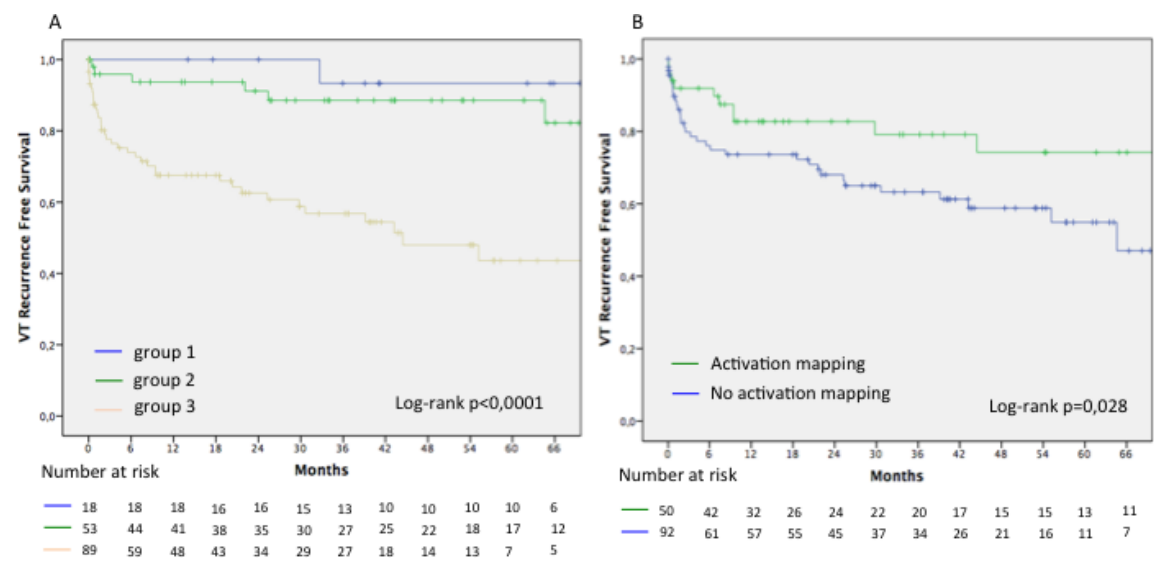

\section{Figure 3.}
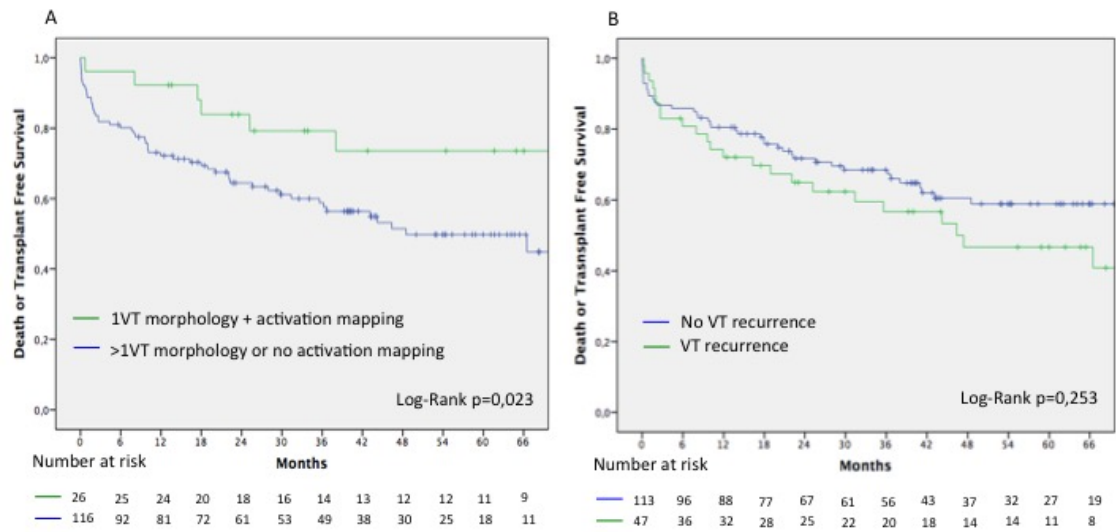

Figure 4.

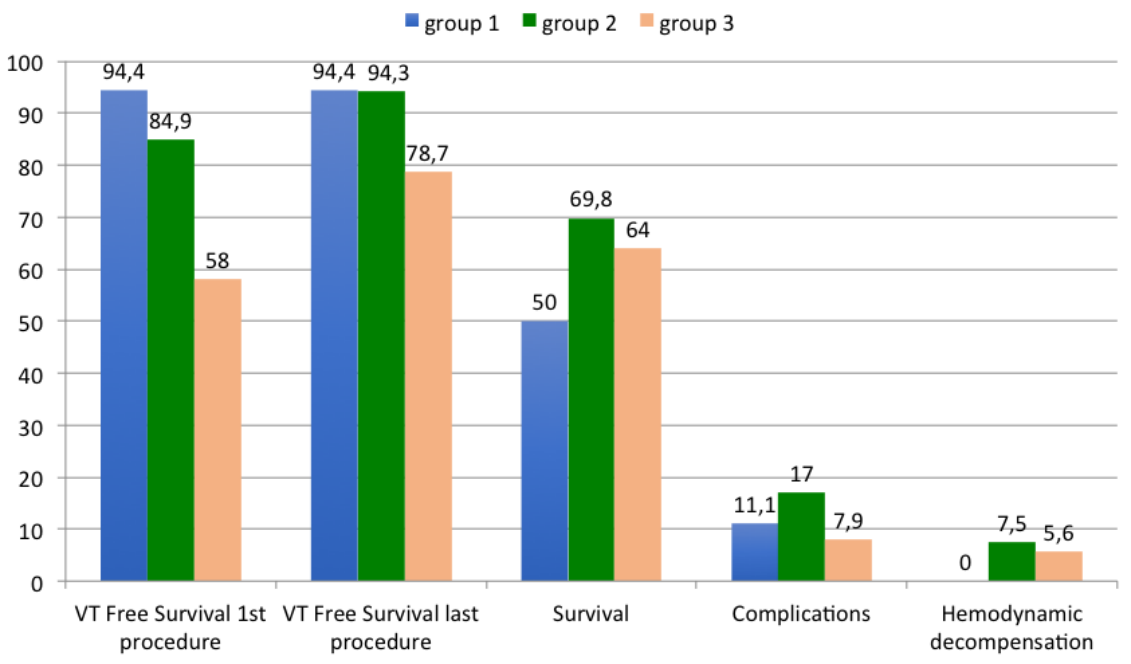


Table 1. Baseline characteristics of the patients

\begin{tabular}{|c|c|c|c|c|c|}
\hline & $\begin{array}{l}\text { Overall } \\
(n=160)\end{array}$ & $\begin{array}{l}\text { Group } 1 \\
(n=18)\end{array}$ & $\begin{array}{l}\text { Group 2 } \\
(n=53)\end{array}$ & $\begin{array}{l}\text { Group } 3 \\
(n=89)\end{array}$ & $P$ value \\
\hline $\begin{array}{l}\text { Age (mean } \pm \\
\text { SD) }\end{array}$ & $63 \pm 13$ & $69 \pm 11$ & $61 \pm 14$ & $62 \pm 13$ & 0.080 \\
\hline Male (n, \%) & $144(90)$ & $14(78)$ & $48(91)$ & $82(92)$ & 0.178 \\
\hline Hypertension & $97(61)$ & $13(72)$ & $32(60)$ & $52(58)$ & 0.550 \\
\hline Diabetes & $44(28)$ & $4(22)$ & $14(26)$ & $26(29)$ & 0.813 \\
\hline Hyperlipidemia & $87(54)$ & $10(56)$ & $27(51)$ & $50(56)$ & 0.828 \\
\hline BMI & $27.2 \pm 4,3$ & $26.9 \pm 4,2$ & $26.9 \pm 3,8$ & $27.4 \pm 4,5$ & 0.779 \\
\hline NYHA class - I - & $35(22) 98(61)$ & $2(11) 11(61) 5$ & $14(26) 32(60) 4$ & $19(21) 55(62)$ & 0.063 \\
\hline II - III - IV & $24(15) 3(2)$ & $(28)-$ & (8) $3(6)$ & $15(17)-$ & \\
\hline $\begin{array}{l}\text { Chronic renal } \\
\text { disease }\end{array}$ & $44(28)$ & $6(33)$ & $15(28)$ & $23(26)$ & 0.800 \\
\hline $\begin{array}{l}\text { Prior } \\
\text { myocardial } \\
\text { infarction }\end{array}$ & $80(50)$ & $7(39)$ & $26(49)$ & $47(53)$ & 0.552 \\
\hline $\begin{array}{l}\text { Prior } \\
\text { revascularization }\end{array}$ & $62(39)$ & $2(11)$ & $23(43)$ & $37(42)$ & 0.037 \\
\hline Prior stroke & $13(8)$ & $1(6)$ & $1(2)$ & $11(12)$ & 0.080 \\
\hline LVEF & $35 \pm 15$ & $41 \pm 16$ & $40 \pm 17$ & $31 \pm 12$ & $<0.0001$ \\
\hline Betablocker & $122(76)$ & $12(67)$ & $40(76)$ & $70(79)$ & 0.545 \\
\hline ACE-I or ARB & $116(73)$ & $13(72)$ & $36(68)$ & $69(78)$ & 0.733 \\
\hline Sotalol & $5(3)$ & - & $2(4)$ & $3(3)$ & 0.715 \\
\hline $\begin{array}{l}\text { Calcium } \\
\text { cannel blocker }\end{array}$ & $17(11)$ & $3(17)$ & $5(9)$ & $9(10)$ & 0.672 \\
\hline Amiodarone & $65(41)$ & $6(33)$ & $23(43)$ & $36(40)$ & 0.753 \\
\hline Anticoagulation & $70(44)$ & $8(44)$ & $19(36)$ & $43(48)$ & 0.363 \\
\hline $\begin{array}{l}\text { Underlying heart } \\
\text { disease - } \\
\text { ischaemic - } \\
\text { non-ischaemic } \\
\text { cardiomyopathy }\end{array}$ & $93(58) 67(42)$ & $9(50) 9(50)$ & $30(56) 23(44)$ & $54(61) 35(39)$ & 0.117 \\
\hline $\begin{array}{l}\text { Arrhythmic } \\
\text { storm (n, \%) }\end{array}$ & $39(24)$ & $4(22)$ & $11(21)$ & $24(27)$ & 0.689 \\
\hline $\begin{array}{l}\text { PAINSDD } \\
\text { score (mean } \pm \\
\text { SD) }\end{array}$ & $11 \pm 7$ & $11 \pm 6$ & $10 \pm 7$ & $11 \pm 7$ & 0.410 \\
\hline
\end{tabular}

BMI: body mass index; NYHA: New York Heart Association functional class; LVEF: left ventricular ejection fraction; ACE-I: Angiotensin-converting-enzyme inhibitors; ARB: Angiotensin-receptor blockers.

Table 2. Procedural data.

\begin{tabular}{|c|c|c|c|c|c|}
\hline & $\begin{array}{l}\text { Overall } \\
(n=160)\end{array}$ & $\begin{array}{l}\text { Group } 1 \\
(n=18)\end{array}$ & $\begin{array}{l}\text { Group 2 } \\
(n=53)\end{array}$ & $\begin{array}{l}\text { Group 3 } \\
(\mathrm{n}=89)\end{array}$ & $P$ value \\
\hline $\begin{array}{l}\text { Number of } \\
\text { procedures (n, } \\
\%)-1-2-3-4\end{array}$ & $\begin{array}{l}129(80) 25(16) \\
3(2) 3(2)\end{array}$ & $17(94) 1(6)--$ & $\begin{array}{l}46(87) 6(11)-1 \\
(2)\end{array}$ & $\begin{array}{l}66(74) 18(20) 3 \\
\text { (4) } 2(2)\end{array}$ & 0.327 \\
\hline
\end{tabular}




\begin{tabular}{|c|c|c|c|c|c|}
\hline & $\begin{array}{l}\text { Overall } \\
(n=160)\end{array}$ & $\begin{array}{l}\text { Group } 1 \\
(\mathrm{n}=18)\end{array}$ & $\begin{array}{l}\text { Group } 2 \\
(\mathrm{n}=53)\end{array}$ & $\begin{array}{l}\text { Group } 3 \\
(n=89)\end{array}$ & $P$ value \\
\hline $\begin{array}{l}\text { Procedure } \\
\text { time (mean } \pm \\
\text { SD) }\end{array}$ & $196 \pm 56$ & $137 \pm 39$ & $188 \pm 52$ & $212 \pm 53$ & $<0.0001$ \\
\hline $\begin{array}{l}\text { Fluoroscopy } \\
\text { time (mean } \pm \\
\text { SD) }\end{array}$ & $6.7 \pm 6.3$ & $6.4 \pm 7.8$ & $6.5 \pm 5.5$ & $6.8 \pm 6.6$ & 0.959 \\
\hline $\begin{array}{l}\text { Radiation dose } \\
\left(\mathrm{cGy}^{*} \mathrm{~cm}^{2}\right) \\
(\text { mean } \pm \mathrm{SD})\end{array}$ & $219 \pm 337$ & $237 \pm 518$ & $208 \pm 305$ & $221 \pm 320$ & 0.962 \\
\hline $\begin{array}{l}\text { LV access }(\mathrm{n}, \%) \\
\text { - transseptal } \\
\text { only - retroaortic } \\
\text { only - } \\
\text { transseptal + } \\
\text { retroaortic }\end{array}$ & $\begin{array}{l}121(76) 22(14) \\
5(3)\end{array}$ & $\begin{array}{l}12(67) 2(11) 1 \\
(6)\end{array}$ & $37(70) 11(21)-$ & $\begin{array}{l}72(81) 9(10) 4 \\
(5)\end{array}$ & 0.148 \\
\hline $\begin{array}{l}\text { RV mapping } \\
(\mathrm{n}, \%)\end{array}$ & $49(31)$ & $2(11)$ & $22(42)$ & $25(28)$ & 0.040 \\
\hline $\begin{array}{l}\text { Multielectrode } \\
\text { mapping } \\
\text { catheter (n, } \\
\%)\end{array}$ & $107(67)$ & $9(50)$ & $33(62)$ & $65(73)$ & 0.114 \\
\hline $\begin{array}{l}\text { Clinical VT } \\
\text { cycle length } \\
(\text { mean } \pm \text { SD) }\end{array}$ & $372 \pm 92$ & $318 \pm 52$ & $381 \pm 103$ & $369 \pm 87$ & 0.393 \\
\hline $\begin{array}{l}\text { Epicardial } \\
\text { access }(n, \%)\end{array}$ & $46(29)$ & $3(17)$ & $14(26)$ & $29(33)$ & 0.402 \\
\hline $\begin{array}{l}\text { Endocardial } \\
\text { scar }(\%)(\text { mean } \\
\pm \mathrm{SD})\end{array}$ & $15.8 \pm 13.1$ & $8.2 \pm 5$ & $12.3 \pm 11.8$ & $18.8 \pm 13.7$ & 0.008 \\
\hline $\begin{array}{l}\text { Epicardial scar } \\
(\%)(\text { mean } \pm \\
\text { SD) }\end{array}$ & $17.7 \pm 25.3$ & - & $24.3 \pm 29.4$ & $15.1 \pm 23.6$ & 0.335 \\
\hline $\begin{array}{l}\text { Radiofrequency } \\
\text { time } \\
(\text { min })(\text { mean } \pm \\
\text { SD })\end{array}$ & $21.9 \pm 13.2$ & $13.8 \pm 6.4$ & $19.1 \pm 10.1$ & $23.7 \pm 14.5$ & 0.215 \\
\hline $\begin{array}{l}\text { PVS at the } \\
\text { end of } \\
\text { procedure (n, } \\
\%)\end{array}$ & $129(81)$ & $7(39)$ & $44(83)$ & $78(88)$ & $<0.0001$ \\
\hline
\end{tabular}




\begin{tabular}{|c|c|c|c|c|c|}
\hline & $\begin{array}{l}\text { Overall } \\
(n=160)\end{array}$ & $\begin{array}{l}\text { Group } 1 \\
(n=18)\end{array}$ & $\begin{array}{l}\text { Group 2 } \\
(\mathrm{n}=53)\end{array}$ & $\begin{array}{l}\text { Group 3 } \\
(n=89)\end{array}$ & $P$ value \\
\hline $\begin{array}{l}\text { Major } \\
\text { complications - } \\
\text { Vascular - } \\
\text { Tamponade - } \\
\text { Stroke - Acute } \\
\text { hemodynamic } \\
\text { decompensation } \\
\text { - Coronary } \\
\text { oclussion }\end{array}$ & $\begin{array}{l}4(2.5) 4(2.5)-9 \\
(5.6) 1(0.6)\end{array}$ & $\begin{array}{l}1(5.6) 1(5.6)-- \\
-\end{array}$ & $\begin{array}{l}2(3.8) 3(5.7)-4 \\
(7.5)-\end{array}$ & $\begin{array}{l}1(1.1)--5(5.6) \\
1(1.1)\end{array}$ & 0.329 \\
\hline
\end{tabular}

LV: left ventricle; RV: right ventricle; VT: ventricular tachycardia; PVS: programmed ventricular stimulation Table 3. Univariate and multivariate analysis for VT recurrence.

\begin{tabular}{lllll}
\hline Variables & Univariate Model & Univariate Model & Multivariate Model & Multivariate Mc \\
\hline & HR (95\% CI) & P value & HR (95\% CI) & P value \\
Inducibility > 1VT morphology & $10.75(1.47-78.53)$ & 0.019 & $12.05(1.60-90.79)$ & 0.016 \\
Acute success & $0.65(0.36-1.16)$ & 0.150 & $0.62(0.34-1.14)$ & 0.125 \\
Lage Endo/Epi scar $(>30 \%)$ & $2.28(1.20-4.33)$ & 0.011 & $1.71(0.88-3.30)$ & 0.110 \\
LVEF < 30\% & $1.99(1.11-3.57)$ & 0.020 & $1.43(0.78-2.62)$ & 0.243 \\
Activation mapping & $0.47(0.23-0.96)$ & 0.039 & $0.63(0.31-1.30)$ & 0.216 \\
\hline
\end{tabular}

Table 4. Univariate and multivariate analysis for death or tranplant.

\begin{tabular}{lllll}
\hline Variables & Univariate Model & Univariate Model & Multivariate Model & Multivar \\
\hline & HR (95\% CI) & P value & HR (95\% CI) & P value \\
1VT morphology + activation mapping & $2.61(1.12-6.06)$ & 0.026 & $2.08(0.88-4.93)$ & 0.095 \\
LVEF $<30 \%$ & $2.69(1.63-4.21)$ & $<0.0001$ & $2.43(1.45-4.07)$ & 0.001 \\
VT recurrence & $0.74(0.45-1.23)$ & 0.255 & $1.13(0.68-1.87)$ & 0.623 \\
Lage Endo/Epi scar $(>30 \%)$ & $1.20(0.66-2.17)$ & 0.544 & $0.95(0.52-1.74)$ & 0.889 \\
\hline
\end{tabular}

